\title{
BMJ Open Analysis of the challenges in implementing guidelines to prevent the spread of multidrug-resistant gram- negatives in Europe
}

\author{
Evelina Tacconelli, ${ }^{1,2,3}$ Michael Buhl, ${ }^{1}$ Hilary Humphreys, ${ }^{4,5,6}$ Veronika Malek, ${ }^{7}$ \\ Elisabeth Presterl, ${ }^{3,5,8}$ Jesús Rodriguez-Baño, ${ }^{3,9}$ Margreet C Vos, ${ }^{5,10}$ Walter Zingg, ${ }^{11}$ \\ Nico T Mutters, ${ }^{\oplus, 12}$ EUCIC StopNegative group
}

To cite: Tacconelli E, Buhl M, Humphreys $\mathrm{H}$, et al. Analysis of the challenges in implementing guidelines to prevent the spread of multidrug-resistant gramnegatives in Europe. BMJ Open 2019;9:e027683. doi:10.1136/ bmjopen-2018-027683

- Prepublication history and additional material for this paper are available online. To view these files, please visit the journal online (http://dx.doi. org/10.1136/bmjopen-2018027683).

Received 2 November 2018 Revised 11 February 2019 Accepted 5 April 2019
D) Check for updates

(c) Author(s) (or their employer(s)) 2019. Re-use permitted under CC BY-NC. No commercial re-use. See rights and permissions. Published by BMJ.

For numbered affiliations see end of article.

\section{Correspondence to} Dr Nico T Mutters; Nico.Mutters@uniklinik-freiburg. de

\section{ABSTRACT}

Objective The main objective of the study was to investigate major differences among European countries in implementing infection prevention and control (IPC) measures and reasons for reduced compliance. Design An online survey including experts in IPC and a gap analysis were conducted to identify major limitations in implementing IPC guidelines.

Setting Europe.

Main outcome measures Four areas were targeted: (1) healthcare structure, (2) finances, (3) culture and (4) education and awareness. Perceived compliance to IPC measures was classified as low $(<50 \%)$, medium $(50 \%$ to $80 \%$ ) and high (>80\%). Countries were classified in three regions: North-Western Europe (NWE), Eastern Europe (EE) and Southern Europe (SE).

Results In total, 482 respondents from 34 out of 44 (77.3\%) European countries participated. Respondents reported availability of national guidelines to control multidrug-resistant Gram-negatives (MDR-GN) in 20 countries (58.0\%). According to participants, compliance with IPC measures ranged from $17.8 \%$ (screening at discharge) to $96.0 \%$ (contact precautions). Overall, three areas were identified as critical for the compliance rate: (1) number of infection control staff, (2) IPC dedicated educational programmes and (3) number of clinical staff. Analysis of reasons for low compliance showed high heterogeneity among countries: participants from NWE and SE deemed the lack of educational programmes as the most important, while those from EE considered structural reasons, such as insufficient single bed rooms or lacking materials for isolation, as main contributors to the low compliance.

Conclusions Although national guidelines to reduce the spread of MDR-GN are reported in the majority of the European countries, low compliance with IPC measures was commonly reported. Reasons for the low compliance are multifactorial and vary from region to region. Crosscountry actions to reduce the spread of MDR-GN have to consider structural and cultural differences in countries. Locally calibrated interventions may be fruitful in the future.
Strengths and limitations of this study

- By summarising the opinions of almost 500 experts, covering $\sim 75 \%$ of all European countries, this analysis on the implementation of infection prevention and control (IPC) measures is the most comprehensive in Europe, even though some countries were over-represented.

- Combining open online survey, direct emailing and offering an open booth for attendees at the European Congress of Clinical Microbiology and Infectious Diseases conference, this study summarises the opinion of a large range of professionals in the field.

- The length of the survey may have been perceived as an obstacle by IPC professionals, and thus, decreased response rate potentially leading to a bias.

\section{BACKGROUND}

The global increase of healthcare-associated infections (HAI) presents a growing concern in healthcare worldwide. According to the European Centre for Disease Prevention and Control (ECDC), the annual number of HAI exceeds 2.6 million and produces the highest estimated amount of disability-adjusted-life-years, surpassing all other reported communicable diseases in the European Union and European Economic Area. ${ }^{1}$ Multidrug-resistant Gram-negative (MDR-GN) bacteria have become increasingly common as a cause for HAI, such as central line-associated bloodstream infections, wound or surgical site infections and catheter-associated urinary tract infections. ${ }^{2}{ }^{3}$ The US National Healthcare Safety Network identified Gram-negative bacteria as the cause of more than $30 \%$ of HAI overall, with almost $70 \%$ of all infections due to Gram-negatives occurring in the intensive care setting. ${ }^{4}$ These infections significantly increase the risk of short- and long-term mortality and hospital 
costs. ${ }^{5}$ Currently, there is no general consensus on the most effective infection prevention and control (IPC) measures and intervention strategies for controlling the spread of MDR-GN in healthcare facilities. The implementation of IPC measures at a time when antibiotic treatment options are limited, become a particular priority for the control of MDR-GN to ensure patient safety. ${ }^{26}$ Multiple barriers exist for the implementation of IPC measures against MDR-GN, ${ }^{7}$ such as the many different bacterial species involved, the differences in mechanisms of resistance and the varying transmission dynamics. ${ }^{8}$ Although stakeholders such as the ECDC since many years are working together with the European Union and European Economic Area member states to increase awareness to the problem and enforce homogeneity of infection control measures, a common European strategy to deal with the spread of MDR-GN bacteria is not yet achieved. ${ }^{89}$

The European Committee on Infection Control (EUCIC) in close cooperation with the European Society of Clinical Microbiology and Infectious Diseases (ESCMID) study group for nosocomial infections launched the STOP-Negative (Reality and limitationS of InfecTiOn Prevention and control measures against multidrug-resistant Gram-NEGATIVEs) project aiming at investigating major differences among European countries in implementing IPC measures, and understanding reasons for reduced compliance.

\section{METHODS}

\section{Objectives}

The primary objectives of the study were: (1) to investigate major differences among European countries in implementing IPC measures and (2) to identify areas for improvement. Secondary objectives were: (1) to explore differences in defining susceptibility patterns of target MDR-GN bacteria and (2) to check availability of national guidelines targeting MDR-GN bacteria.

\section{Setting and participants}

EUCIC is a standing committee, initiated by ESCMID to support the global efforts of standardising and harmonising IPC measures across Europe, to foster research in IPC and to provide training in IPC. EUCIC has one representative from each country, leading a national committee of 3 to 9 experts. The committee's network now includes $>90 \%$ of all Europe. We developed a 15-item cross-sectional, self-administered online survey. After being piloted among the EUCIC Stop-Negative working group, it was sent to the EUCIC national representatives by email and was made accessible to the general public on the committee's website. Additionally, during the 27th European Congress of Clinical Microbiology and Infectious Diseases in Vienna, Austria, conference participants were invited to fill out the electronic questionnaire at the EUCIC booth.

\section{Survey design, administration and analysis}

The survey was created by the EUCIC Stop-Negative working group (MB, HH, NM, EP, JR-B, ET, MCV, WZ). Questions addressed the availability of country-specific guidelines on the prevention of HAI due to MDR-GN bacteria, and barriers to successful implementation of IPC measures. Participants were asked to select the three most important challenges in their hospital/countries, which were defined as the most important priorities in improving effectiveness and use of guidelines. The questionnaire is included as an appendix in the supplementary material. Collected data was analysed using Microsoft Excel 2010 and IBM SPSS Statistics V.23.

\section{Classification of MDR-GN}

Respondents reporting availability of national or international guidelines in their countries were asked to define, how the target MDR-GN was defined (in terms of susceptibility pattern). For the purpose of this survey, MDR-GN included the following bacteria: carbapenemase-producing Pseudomonas aeruginosa, Acinetobacter baumannii and Klebsiella spp and carbapenem-resistant $P$. aeruginosa, A. baumannii and Klebsiella spp, according to European Committee on Antimicrobial Susceptibility Testing clinical breakpoints.

\section{Perceived compliance with guidelines and gap analysis}

Perceived compliance with IPC measures recommended by the guidelines was classified as low $(<50 \%)$, medium (50\% to $80 \%)$ and high (>80\%). To investigate low and medium levels of compliance respondents were asked to select the main reason for limited compliance from the following options: staff shortage, structural reasons (ie, not enough single bed rooms, not enough material for isolation precautions (ie, gloves and gowns)), financial reasons (ie, not possible to block beds in multi-bedded rooms due to missing reimbursement), cultural reasons/ controversial efficacy (ie, isolation precaution measures are not considered relevant or efficient, MDR-GN pathogens are not considered virulent, corruption impedes implementation of measures, ie, due to cash drain), and educational reasons (ie, healthcare-workers do not know how to perform IPC measures such as isolation precaution measures, proper procedures of wearing and disposing of personal protective equipment). The answers were then stratified by each respondent's country of origin and separated into three geographical regions, adapted from the WHO regions: Eastern Europe (EE), North-Western Europe (NWE) and Southern Europe (SE). Respondents were asked to identify the three most important requirements to improve the effectiveness and/or use of IPC measures. Answers were then compared between the previously introduced geographical regions.

\section{Statistical analyses}

For descriptive purposes, arithmetic mean values with SD, medians with IQR and cumulative frequencies were calculated where appropriate. Two-sided $p$ values of less than 
Table 1 Countries of origin of respondents (alphabetical order)

\begin{tabular}{llll}
\hline Regions & North-Western Europe (n) & Eastern Europe (n) & Southern Europe (n) \\
\hline Countries & Austria (10) & Azerbaijan (1) & Albania (2) \\
& Belgium (2) & Czech Republic (2) & Bosnia and Herzegovina (2) \\
& Denmark (10) & Georgia (2) & Croatia (5) \\
& France (81) & Hungary (4) & Cyprus (5) \\
& Germany (13) & Latvia (1) & Greece (8) \\
& Ireland (18) & Lithuania (1) & Israel (2) \\
& Netherlands (2) & Republic of Moldova (2) & Kosovo (2) \\
& Norway (2) & Romania (197) & Macedonia (1) \\
& Sweden (1) & Slovakia (2) & Portugal (2) \\
& Switzerland (1) & & Spain (28) \\
& UK (5) & & Slovenia (3) \\
\hline Total (\% of total respondents) & $145(30.1 \%)$ & & Turkey (8)
\end{tabular}

0.05 were considered to indicate statistical significance. Statistical analysis was performed using the SPSS statistical package (SPSS V.21.0, Chicago, Illinois). Sample size calculation was performed before distributing the questionnaire. The statistical description of the universe of reference (ie, European clinical microbiology/infectious diseases/infection control specialists) according to geographical region was based on a previous analysis done by ESCMID. ${ }^{10}$ The sample size to attain a confidence level of $95 \%$ and a precision of $\pm 5 \%$ was calculated as a minimum of 400 respondents.

\section{Patient and public involvement}

No patients were involved in the design of the study nor its conduction. Neither were patients the target group of this study. The public will be informed by ESCMID/ EUCIC newsletters on publication of the study. Publication will be in an open-access manner.

\section{RESULTS}

\section{Respondents' characteristics}

In total, 482 respondents $(40.9 \%$ infectious diseases specialists, $29.3 \%$ clinical microbiologists and $24.5 \%$ infection control/hospital hygiene specialists) from 34 European countries (out of 44 countries) took part in the survey (table 1).

\section{Classification of MDR-GN}

The majority of participants reported resistance to carbapenems to be the major classification criterion for all three pathogens (table 2). More than half also considered cephalosporin resistance in combination with carbapenem resistance to classify MDR - P. aeruginosa and Klebsiella spp (61.4\% and $68.2 \%$, respectively). Less than half $(39.2 \%)$ used cephalosporin resistance as an additional classification variable to define MDR A. baumannii. Resistance to aminoglycosides and fluoroquinolones, besides resistance to carbapenems and cephalosporins, were considered by half of the respondents to define multidrug-resistance (table 2).

\section{Guidelines for MDR-GN}

More than half of the respondents reported that either national $(101 ; 58.0 \%)$ or local/regional $(113 ; 64.9 \%)$ guidelines on the prevention of MDR-GN were available in their country. Country guidelines for single organisms, that is for MDR P. aeruginosa, A. baumannii, and Klebsiella spp were reported by one-third of the respondents (27.0\%, 32.4\% and 37.4\%, respectively). National IPC guidelines mainly address the general patient population $(87 ; 60.2 \%)$, rather than focussing on high-risk patients $(37 ; 39.8 \%)$. When they did, the following patient populations were predominantly identified as high-risk: intensive-care unit patients $(135 ; 76.7 \%)$, immuno-compromised patients $(111 ; 63.1 \%)$ and haematology-oncology patients $(110 ; 62.5 \%)$.

\section{Implementation of infection prevention and control measures}

The degree of implementing IPC measures varied significantly among countries. Contact precaution measures and spatial isolation of patients are applied in $96.0 \%$ $(168 / 175)$ and $71.3 \%(124 / 174)$ of the centres, respectively. However, regular universal screening at admission or discharge for all patients is performed in only $21.7 \%$ $(38 / 175)$ and $17.8 \%(31 / 174)$ of the centres, respectively (figure 1). Of those who implemented admission screening, $77.0 \%$ (134/174) screen high-risk patients and $21.7 \%(38 / 175)$ screen all patients at admission. Selective digestive decolonisation of MDR-GN high-risk patients was reported by $30.9 \%$ (54/175). Details about drugs and 
Table 2 Antibiotics included in the definition of multidrug-resistance for Pseudomonas aeruginosa, Acinetobacter baumannii and Klebsiella pneumoniae

\begin{tabular}{|c|c|c|c|}
\hline Antimicrobial agents & Pseudomonas aeruginosa, $\mathrm{n}(\%)$ & $\begin{array}{l}\text { Acinetobacter baumannii, } \mathrm{n} \\
(\%)\end{array}$ & Klebsiella spp, n (\%) \\
\hline Penicillins + beta-lactamase inhibitors & $82(46.6)$ & $64(36.4)$ & 76 (43.2) \\
\hline Cephalosporins & $108(61.4)$ & $69(39.2)$ & $120(68.2)$ \\
\hline Carbapenems & $149(84.7)$ & $147(83.5)$ & $142(80.7)$ \\
\hline Fluoroquinolones & $92(52.3)$ & $82(46.6)$ & 87 (49.9) \\
\hline Aminoglycosides & 79 (44.9) & $73(41.5)$ & $77(43.8)$ \\
\hline Monobactams & 25 (14.2) & NA & 29 (16.5) \\
\hline Phosphonic acid & $7(4.0)$ & NA & $6(3.4)$ \\
\hline Polymyxins & $43(24.4)$ & $50(28.4)$ & $45(25.6)$ \\
\hline Tetracyclines & NA & $20(11.4)$ & $21(11.9)$ \\
\hline Trimethoprim/sulfamethoxazole & NA & $29(16.5)$ & $25(14.2)$ \\
\hline Cephamycins & NA & NA & $28(15.9)$ \\
\hline Glycylcyclines & NA & NA & 24 (13.6) \\
\hline Other & $3(1.7)$ & $7(4.0)$ & $4(2.3)$ \\
\hline Total & $176(100)$ & $176(100)$ & $176(100)$ \\
\hline
\end{tabular}

NA, not applicable.

modalities were not asked. General antimicrobial stewardship programmes were reported by $73.4 \%$ (126/171) and programmes specifically targeting high-risk patients by $25.7 \%(28 / 109)$ of the respondents.

Perceived compliance with IPC measures

Highest compliance was reported for electronic flagging of patients colonised or infected with MDR-GN, enhanced environmental cleaning, and different (admission, discharge, periodical) forms of screening modalities. On the other hand, lowest compliance was reported for staff cohorting and patient isolation in multi-bedded rooms while blocking neighbouring beds (figure 2).

Main reasons for perceived low compliance

Reasons reported for low compliance varied across countries and regions (figure 3). NWE and SE indicated lack of appropriate IPC education for perceived low compliance

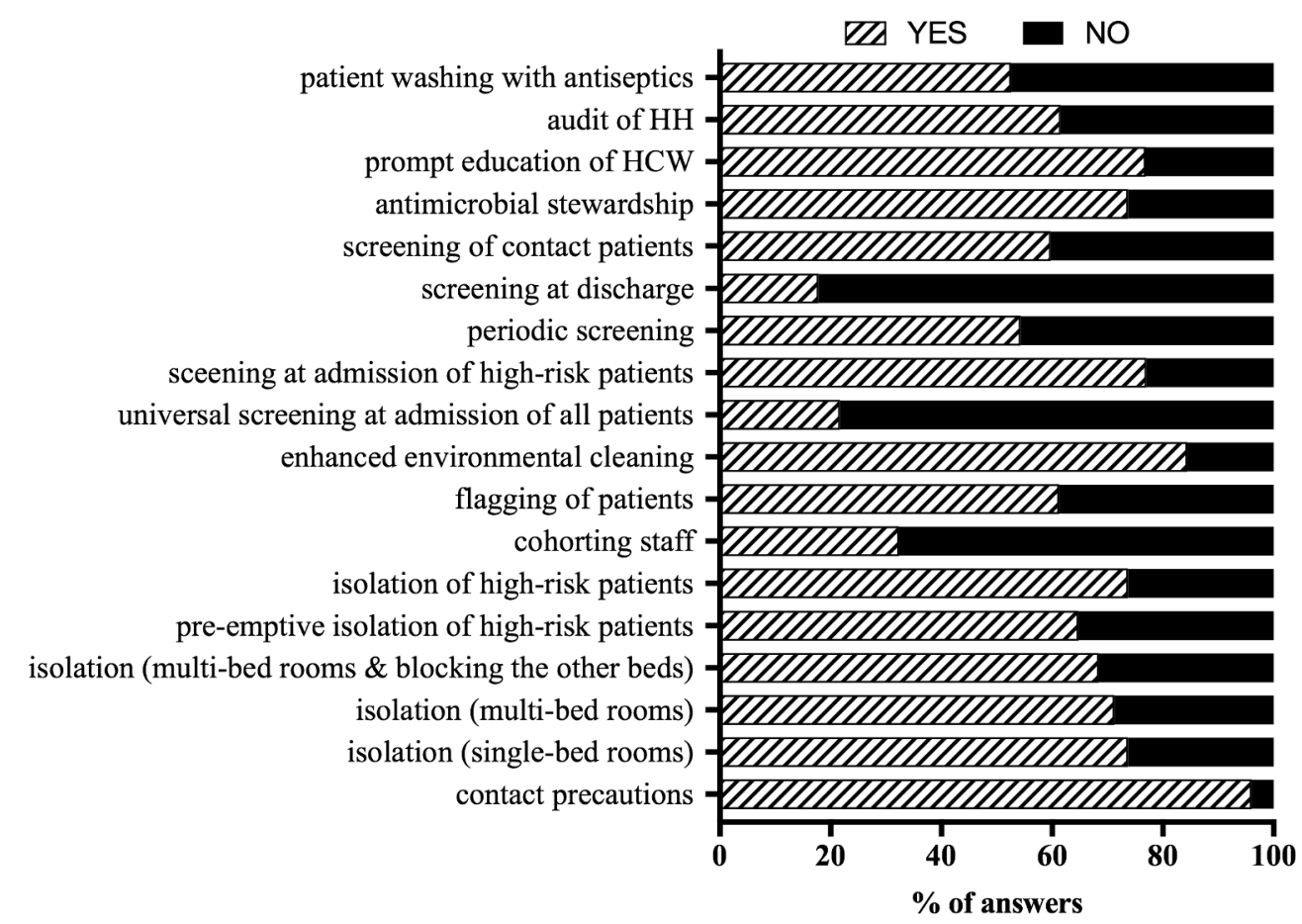

Figure 1 Percentage of implementation of IPC measures to reduce the spread of MDR-GN among survey respondents. HCW, healthcare worker; HH, hand hygiene; IPC, infection prevention and control; MDR-GN, multidrug-resistant Gram-negatives. 


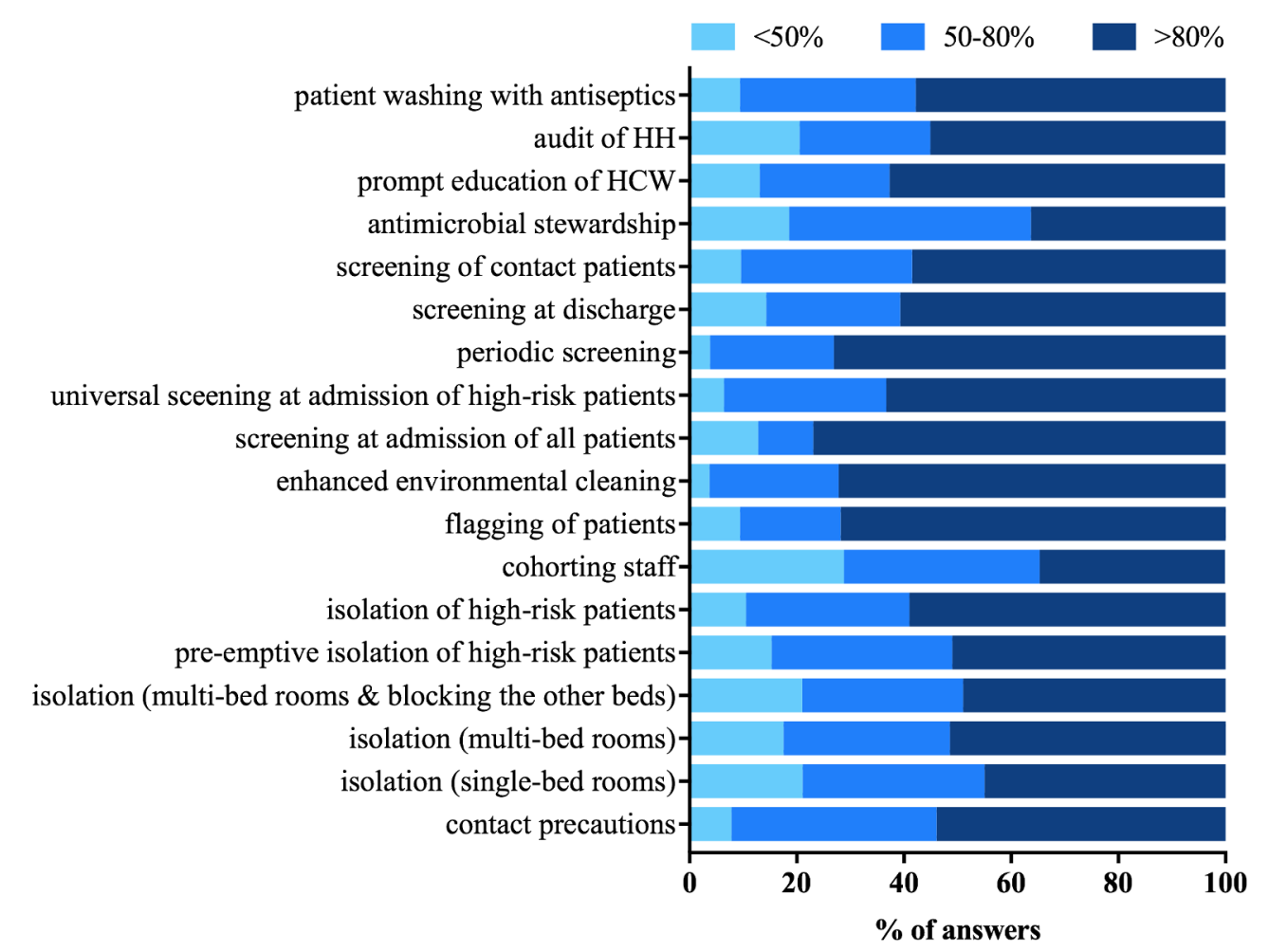

Figure 2 Perceived level of compliance with IPC measures to reduce the spread of MDR-GN among survey respondents. $\mathrm{HCW}$, healthcare worker; $\mathrm{HH}$, hand hygiene; IPC, infection prevention and control; MDR-GN, multidrug-resistant Gramnegatives.

with staff cohorting ( $76.5 \%$ in NWE vs $91.7 \%$ in SE). In EE on the other hand, staff shortage was the main reason for perceived low compliance with staff cohorting $(81.0 \%)$. In $\mathrm{EE}$, financial restraints were the major challenge for high compliance with screening measures $(62.0 \%)$, while SE and NWE indicated cultural reasons and staff shortage as main contributors. Budget restrictions were also reported as main reasons for perceived low compliance with isolation precaution measures in NWE and SE $(72.5 \%$ and $54.7 \%$, respectively), while structural reasons (lack of single rooms) were the main challenge for applying isolation precaution measures in EE $(75.0 \%)$. Lack of antimicrobial stewardship programmes were overall mainly linked to educational issues (45.5\% in NWE, $53.0 \%$ in EE and $40.0 \%$ in SE) (figure 3).

\section{Areas for improvement}

Figure 4 summarises suggested areas for improvement. Increased IPC staffing (nurses and doctors) in a situation with trained staff shortage was cited the most important need for IPC improvement in EE and SE $(78.4 \%$ and $57.9 \%$, respectively), while adequate financial resources for IPC staffing (nurses and doctors) $(50.7 \%)$ and clinical staffing (nurses and doctors) $(55.1 \%)$ was mentioned in NWE. All respondents selected educational programmes and training frontline staff as an important area for improvement $49.3 \%$ in NWE, $40.4 \%$ in SE and $44.6 \%$ in $\mathrm{EE}$ ). Particularly in SE, a change in the political awareness with regards to healthcare management was mentioned as another significant need $(49.1 \%)$. Interestingly, new hospital facilities (31.9\% in NWE, 21.1\% in SE and $13.5 \%$ in $\mathrm{EE})$ and specific legislation (13.0\% in NWE, $12.3 \%$ in $\mathrm{SE}$ and $18.9 \%$ in $\mathrm{EE}$ ) were mentioned less frequently by the respondents.

\section{DISCUSSION}

The rise in MDR-GN has prompted healthcare professionals worldwide to define IPC measures for controlling MDR-GN spread, which need to be implemented at local, national and international levels. ${ }^{11}$ With a response rate covering $77.3 \%$ of all European countries and a number of participants exceeding the calculated number necessary to obtain confidence and precision, we reached representative coverage of all Europe.

The primary objectives of our study were to investigate major differences among European countries in implementing IPC measures and reasons for reduced compliance, as well as to identify areas for improvement. Our results show high heterogeneity of compliance for both, countries and IPC measures. Participants from NWE and SE ranked educational programmes to be most important for improving compliance with IPC measures. Respondents from EE reported structural reasons, such as insufficient single bed rooms or lacking materials for isolation, to be the main contributors to low compliance. Certainly, individual country (or even regional) characteristics need to be taken into consideration when implementing IPC practices. $^{12} 13$ 

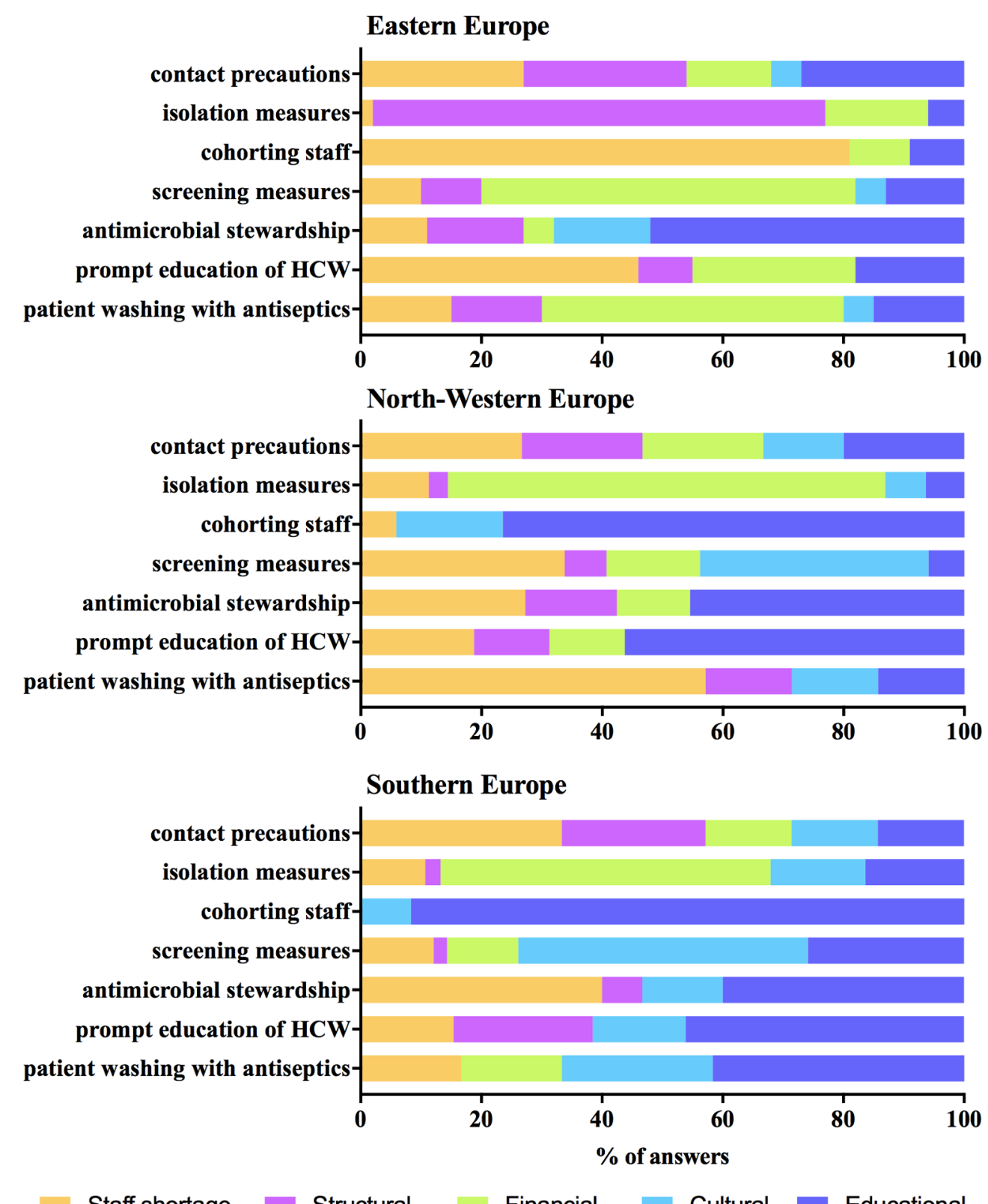

Figure 3 Main reasons for low compliance. HCW, healthcare worker.

Three areas were identified by respondents as critical for compliance: (1) (sufficient) number of infection control staff, (2) IPC dedicated educational programmes and (3) (sufficient) number of clinical staff. Not surprisingly, regional differences also applied to these critical areas for improvement of IPC measures. An overwhelming majority of the respondents from EE, where trained health professionals leave the countries, selected an increase in IPC staff as the most important area for improvement. This was also the most frequent response given by SE respondents. On the contrary, NWE respondents considered an increase in actual clinical staff (nurses and doctors) more important. More IPC staff and establishing educational training programmes were considered less important by NWE respondents. Some differences between regions, however, might be due to other underlying differences in hospital and personnel structure that were not assessed by our survey. For example NWE and SE had lower perceived compliance with cohorting staff compared with the other regions. However, this might be due to higher availability of more individual rooms or a better staffto-patient ratio, and therefore less need for cohorting.

Interestingly, the measures most frequently promoted by guidelines were not the ones with the highest level of compliance; where recommended, contact precaution measures had lower levels of compliance compared with universal screening of patients at admission, although contact precaution measures are obviously far more often applied than screening. Where screening is recommended, however, it appears that hospitals are more willing to implement it. The fact that some measures are perceived more important (or easier to perform) than 


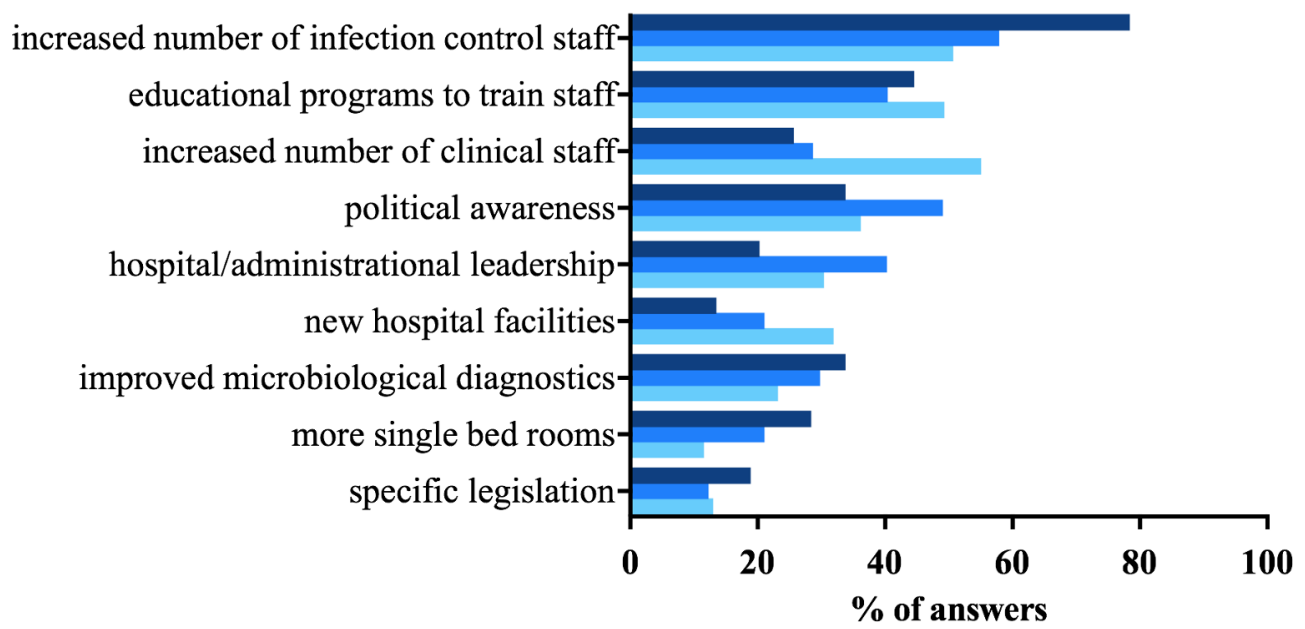

Figure 4 Most important areas for improving compliance with IPC measures to reduce the spread of MDR-GN stratified by EU regions. EU, European Union; IPC, infection prevention and control; MDR-GN, multidrug-resistant Gram-negatives.

others is most likely influenced by both knowledge and personal views. This example shows that implementation does not necessarily follow the level of evidence or the rationale behind a recommendation. While applying contact precaution measures can directly disrupt the transmission of pathogens, screening does not change transmission without being linked to action, such as pre-emptive or subsequent contact or barrier precaution measures. Indeed, these results underline the importance of education, both pre- and postgraduate, in streamlining efforts to prevent the spread of MDR-GN. Therefore, stronger international collaboration is needed, focussing on validating and agreeing with the most effective IPC prevention strategies. In addition to providing evidencebased guidelines for all countries, implementation strategies should be evaluated for their applicability taking into account local organisational and socio-economical contexts.

The secondary outcomes of our study were the exploration of differences in the definition of susceptibility patterns for classifying MDR-GN bacteria. The majority of participants in our survey reported that resistance to carbapenems alone was the major classification criterion for MDR in Gram-negative bacteria. Over half of the respondents also considered cephalosporin resistance in combination with carbapenem resistance for classifying MDR - $P$. aeruginosa and MDR - Klebsiella spp. Resistance to aminoglycosides and fluoroquinolones, besides carbapenem- and cephalosporin resistance, were taken into account by half of the respondents in the definition of multidrug-resistance. Overall, our results show unexpected heterogeneity in classifying of MDR-GN in Europe. This is surprising after ECDC published definitions on multidrug-resistant, extensively drug-resistant and pandrug-resistant pathogens. ${ }^{14}$ The fact that no internationally published recommendation on MDR-GN classification was able to override regional classification systems, complicates transnational communication and finally the control on the spread of MDR-GN. Apparently, standardisation not only is required on the IPC level, but also on the microbiology level. Although efforts to standardise antimicrobial susceptibility testing were largely successful in Europe, and worldwide, efforts to standardise the classification of MDR in Gram-negative bacteria are needed.

Finally, the study aimed to evaluate availability of national guidelines targeting MDR-GN bacteria. According to our survey, only half of the respondents indicated that national guidelines for MDR-GN exist in their countries. This highlights the urgency to develop and implement IPC guidelines contextualised to national situations that are currently missing. The external validity of the results need to be considered behind the background of local epidemiology and country-specific needs that might differ from the overall results.

Our study has limitations. First, since this voluntary survey was comprised of self-selected participants, the opinions might not be entirely representative for a country. Second, some countries are overrepresented, which may have resulted in a so-called 'country-effect', where responses from one country have a disproportionally large effect on the overall position of the region. This holds true for an overrepresentation of Romania for EE (table 1). Additionally, we did not take into account high or low endemicity settings, which might influence screening decisions and compliance with such measures, since screening is likely to depend on perceived population prevalence, and low application may not be interpreted as non-compliance. Third, the elements for compliance depicted in figure 1 might not be necessarily advised in all countries. Participants, however, were invited to comment and feel that the introduction of bias regarding to this might be negligible. 


\section{CONCLUSIONS}

Our findings revealed high heterogeneity in European countries in terms of implementation of IPC measures to reduce the spread of MDR-GN. Low compliance with recommendations has different reasons, which are strongly linked to national and regional contexts. Thus, implementation strategies for IPC need to take into account culture, educational and socio-economical differences and financial capacities among European countries. The spread of MDR-GN is a global health challenge that requires a joint international effort. Our results provide guidance for identification of areas of improvement with regards to international collaboration, which may help to plan joint interventions in Europe to contain the spread of MDR-GN.

\section{Author affiliations}

${ }^{1}$ Infectious Diseases, University Hospital Tübingen, Tübingen, Germany

${ }^{2}$ Infectious Diseases, Department of Diagnostic and Public Health, University of

Verona, Verona, Italy

${ }^{3}$ European Committee on Infection Control, Basel, Switzerland

${ }^{4}$ Royal College of Surgeons in Ireland, Dublin, Ireland

${ }^{5}$ ESCMID Study group for nosocomial infections, Basel, Switzerland

${ }^{6}$ Beaumont Hospital, Dublin, Ireland

${ }^{7}$ Centre of Infectious Diseases, Heidelberg University Hospital, Heidelberg, Germany

${ }^{8}$ Department of Infection Control and Hospital Epidemiology, Medical University of

Vienna, Vienna, Austria

${ }^{9}$ Hospital Universitario Virgen Macarena, Seville, Spain

${ }^{10}$ Department of Medical Microbiology and Infectious diseases, Erasmus MC

Medical Center Rotterdam, Rotterdam, The Netherlands

${ }^{11}$ Infection Control Programme, University of Geneva Hospitals, Geneva, Switzerland

${ }^{12}$ Institute for Infection Prevention and Hospital Epidemiology, Medical Center -

University of Freiburg, Freiburg, Germany

Collaborators Azerbaijan: Professor Akiv Gurbanov, Bulgaria: Professor Rossitza Vatcheva-Dobrevska, Croatia: Professor Rok Civljak, Croatia: Dr Edita Susic, Cyprus: Professor Georgios I Petrikkos and Dr Constantinos Tsioutis, Denmark: Lisbeth Kyndi Bergen, France: Professor Vincent Pascal Jarlier, Greece: Professor Athanassios Tsakris, Hungary: Dr Gyula Prinz, Israel: Professor Yehuda Carmeli, Italy: Professor Claudio Viscoli, Kosovo: Dr Lul Raka, Norway: Dr Per Espen Akselsen, Ireland: Dr Kirsten Schaffer, Romania: Dr Oana Sândulescu, Sweden: Dr Anders Johansson. The EUCIC StopNegative group listed as collaboration group members contributed to data collection.

Contributors ET, MB, HH, VM, EP, JR-B, MCV, WZ, NM contributed to data collection and data analysis. ET, MB, HH, EP, JR-B, MCV, WZ, NM designed the questions for the gap analysis and the survey. NM and ET wrote the first draft of the manuscript, all other authors contributed to the writing of the manuscript. NM, ET and VM analysed the data. All authors contributed to the interpretation of data.

Funding The study was partly funded (NTM) by a grant by the Baden-Württemberg Ministry of Science, Research and the Arts (MWK; grant: "Surveillance von Mehrfach-Antibiotika-Resistenzen"). The study was furthermore non-financially supported by ESCMIDs European Committee on Infection Control. The open-access fee was covered by ESCMID.

Disclaimer The authors would like to express their gratitude to all participants, especially the EUCIC Advisory board and national committee members.
Competing interests JRB has received personal fees from Merck, personal fees from AstraZeneca and grants from Innovative Medicines Initiative, however, all not related to the submitted work. The other authors have nothing to declare.

Patient consent for publication Not applicable.

Provenance and peer review Not commissioned; externally peer reviewed.

Data sharing statement The data is not publicly available. Please contact the corresponding author for further information.

Open access This is an open access article distributed in accordance with the Creative Commons Attribution Non Commercial (CC BY-NC 4.0) license, which permits others to distribute, remix, adapt, build upon this work non-commercially, and license their derivative works on different terms, provided the original work is properly cited, appropriate credit is given, any changes made indicated, and the use is non-commercial. See: http://creativecommons.org/licenses/by-nc/4.0/.

\section{REFERENCES}

1. Cassini A, Plachouras D, Eckmanns T, et al. Burden of six healthcareassociated infections on european population health: estimating incidence-based disability-adjusted life years through a population prevalence-based modelling study. PLoS Med 2016;13:e1002150.

2. Tacconelli E, Cataldo MA, Dancer SJ, et al. ESCMID guidelines for the management of the infection control measures to reduce transmission of multidrug-resistant Gram-negative bacteria in hospitalized patients. Clin Microbiol Infect 2014;20(Suppl 1):1-55.

3. Thaden JT, Li Y, Ruffin F, et al. Increased costs associated with bloodstream infections caused by multidrug-resistant gram-negative bacteria are due primarily to patients with hospital-acquired infections. Antimicrob Agents Chemother 2017;61.

4. Peleg AY, Hooper DC. Hospital-acquired infections due to gramnegative bacteria. N Engl J Med 2010;362:1804-13.

5. Nelson RE, Slayton RB, Stevens VW, et al. Attributable mortality of healthcare-associated infections due to multidrug-resistant gramnegative bacteria and methicillin-resistant staphylococcus aureus. Infect Control Hosp Epidemiol 2017;38:848-56.

6. Allegranzi B, Kilpatrick C, Storr J, et al. Global infection prevention and control priorities 2018-22: a call for action. Lancet Glob Health 2017;5:e1178-e1180.

7. Rodríguez-Baño J, del Toro MD, López-Méndez J, et al. Minimum requirements in infection control. Clin Microbiol Infect 2015;21:1072-6.

8. Otter JA, Mutters NT, Tacconelli E, et al. Controversies in guidelines for the control of multidrug-resistant Gram-negative bacteria in EU countries. Clin Microbiol Infect 2015;21:1057-66.

9. Mutters NT, Tacconelli E. Infection prevention and control in Europe the picture in the mosaic. Clin Microbiol Infect 2015;21:1045-6.

10. Tacconelli E, Poljak M, Cacace M, et al. Science without meritocracy. Discrimination among European specialists in infectious diseases and clinical microbiology: a questionnaire survey. BMJ Open 2012;2:e001993.

11. Price L, MacDonald J, Melone L, et al. Effectiveness of national and subnational infection prevention and control interventions in highincome and upper-middle-income countries: a systematic review. Lancet Infect Dis 2018;18.

12. Dettenkofer M, Ammon A, Astagneau P, et al. Infection control--a European research perspective for the next decade. J Hosp Infect 2011;77:7-10.

13. Gysin DV, Cookson B, Saenz $\mathrm{H}$, et al. Variability in contact precautions to control the nosocomial spread of multi-drug resistant organisms in the endemic setting: a multinational cross-sectional survey. Antimicrob Resist Infect Control 2018;7:81.

14. Magiorakos AP, Srinivasan A, Carey RB, et al. Multidrug-resistant, extensively drug-resistant and pandrug-resistant bacteria: an international expert proposal for interim standard definitions for acquired resistance. Clin Microbiol Infect 2012;18:268-81. 